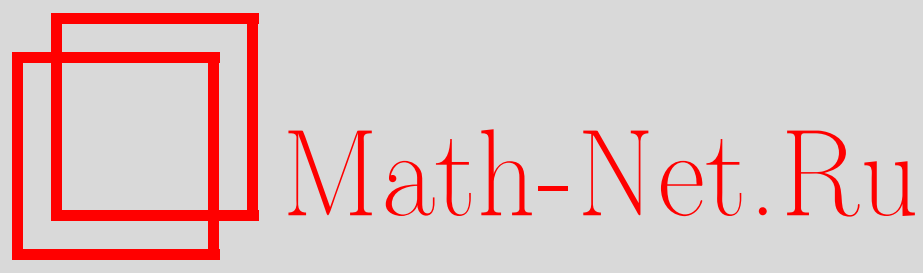

В. С. Погосян, В. Б. Приезжев, Фундаментальные константы в теории двумерных покрывающих деревьев, TMФ, 2016, том 187, номер 3, 580-594

DOI: https://doi.org/10.4213/tmf9136

Использование Общероссийского математического портала Math-Net.Ru подразумевает, что вы прочитали и согласны с пользовательским соглашением http://www . mathnet.ru/rus/agreement

Параметры загрузки:

IP: 52.205 .19 .152

26 апреля 2023 г., 13:41:47

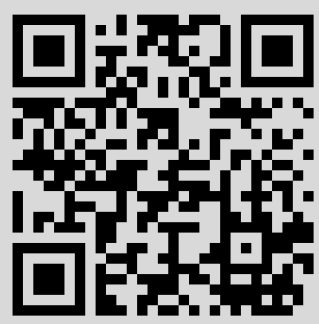




\section{ФУНДАМЕНТАЛЬНЫЕ КОНСТАНТЫ В ТЕОРИИ ДВУМЕРНЫХ ПОКРЫВАЮЩИХ ДЕРЕВЬЕВ}

Три характеристики двумерных покрывающих деревьев связаны между собой нетривиальными соотношениями: средняя высота в модели "песочной горки", петлевая константа квадратной решетки и вероятность возврата случайного блуждания со стертыми петлями. Кратко прослежена долгая история вывода их неожиданных рациональных значений.

Ключевые слова: абелева модель “песочной горки", случайные блуждания со стертыми петлями, модель мономеров-димеров.

DOI: $10.4213 / \operatorname{tmf} 9136$

\section{1. ВВЕДЕНИЕ}

Совершенно различные, на первый взгляд, объекты - модель димеров, случайные блуждания со стертыми петлями (loop-erased random walk (LERW)) и абелева модель "песочной горки" (Abelian sandpile model (ASM)) - тесно связаны между собой, поскольку все они допускают альтернативную формулировку в терминах двух объединяющих концепций: однородных покрывающих деревьев (uniform spanning trees (UST)) и однородных покрывающих унициклов (uniform spanning unicycles (USU)).

На конечном графе $G$ покрывающее дерево - это связное множество ребер, которое не содержит циклов и покрывает все вершины графа $G$. Если множество вершин графа $G$ совпадает с конечным подмножеством $\mathbb{Z}^{2}$, говорят, что покрывающее дерево определено на квадратной подрешетке. UST - это покрывающий подграф графа $G$, который является случайно выбранным деревом с однородной вероятностью. Определение UST, данное для конечных графов, может быть распространено на все подмножество $\mathbb{Z}^{2}$ [1]. Уницикл - это связный граф с одинаковым числом вершин и ребер. USU графа $G$ - это покрывающий подграф графа $G$, являющийся случайно

В. Б. Приезжев благодарен фонду РФФИ за поддержку (грант № 16-02-00252), В. С. Погосян благодарен программе ОИЯИ "Смородинский - Тер-Антонян".

* Институт проблем информатики и автоматизации, НАН Армении, Ереван, Армения

$\dagger$ †аборатория теоретической физики им. Н.Н. Боголюбова, Объединенный институт ядерных исследований, Дубна, Московская обл., Россия. E-mail: vpoghos@theor.jinr.ru, priezzvb@theor.jinr.ru 
выбранным унициклом [2]. Перечисление покрывающих деревьев осуществляется с помощью теоремы Кирхгофа, которая является мощным математическим инструментом для решения различных комбинаторных проблем теоретической физики [3]. Для унициклов подобная теорема отсутствует.

Модель димеров была представлена в 1937 г. Фаулером и Рашбруком [4], но первые точные результаты были получены Кастеляйном, Фишером, Темперли и Стивенсоном [5]-[9] значительно позже, в шестидесятые годы прошлого века. Модель димеров, известная также как проблема плотной упаковки, стала снова популярной в последнее десятилетие [10]. Соответствие между плотной упаковкой димеров и покрывающими деревьями на квадратной решетке было установлено Темперли [11] и распространено на произвольные планарные графы Бартоном и Пемантле [12]. Это соответствие сохраняется при наличии мономеров и ведет в этом случае к моделям покрывающих сетей [13], [14].

Модель LERW определена Лоулером в работе [15] как система путей на решетке, генерируемых простым симметричным случайным блужданием с условием удаления всех замкнутых петель сразу после их возникновения. Формальное определение LERW можно дать следующим образом. Рассмотрим конечную квадратную решетку с множеством вершин $V$ и множеством ребер $E$. Выберем подмножество вершин $W(W \subset V)$, называемое множеством поглощающих вершин. Пусть $\mathcal{P}=\left[u_{0}, u_{1}, u_{2}, \ldots, u_{n}\right]$ - это набор вершин, принадлежащих траектории случайного блуждания, состоящего из $n$ шагов по решетке с началом в точке $u_{0}$ и с концом в одной из поглощающих точек $u_{n} \in W$. Стирание петель в траектории $\mathcal{P}$, обозначаемое как $\operatorname{LE}(\mathcal{P})=\left[\gamma_{0}, \gamma_{1}, \gamma_{2}, \ldots, \gamma_{m}\right]$, состоит в удалении каждой петли в $\mathcal{P}$ в хронологическом порядке. Заметим, что порядок удаления петель фиксирован, поскольку петли удаляются немедленно после их возникновения по мере продолжения траектории $\mathcal{P}$. Упорядоченное множество вершин $\operatorname{LE}(\mathcal{P})$ называется случайным блужданием со стертыми петлями (LERW) на квадратной решетке с множеством поглощающих вершин $W$. Пропп и Вильсон [16] предложили алгоритм, позволяющий генерировать ансамбль UST с помощью LERW. Связь между LERW и покрывающими деревьями обсуждалась в ряде работ [1], [17]-[20], где было показано, что вероятностная мера на LERW совпадает с однородной мерой так называемых химических путей на покрывающих деревьях.

Наконец, ASM - это открытая стохастическая динамическая система, являющаяся нелинейным диффузионным процессом [21], [22]. ASM определяется в терминах переменных высот, приписываемых каждому узлу двумерной квадратной решетки. Высота $h$ принимает четыре целых значения $1,2,3,4$, но лишь некоторая доля всех возможных конфигураций высот, называемых рекуррентными конфигурациями, остается в результате длительного динамического процесса. При больших временах состояние модели характеризуется стационарной мерой, определенной на рекуррентных конфигурациях. Эта мера однородна на рекуррентных конфигурациях и обращается в нуль на переходных конфигурациях. Связь с покрывающими деревьями задается алгоритмом сжигания [23], который устанавливает однозначное соответствие между рекуррентными конфигурациями ASM и покрывающими деревьями на той же решетке.

Все упомянутые модели обладают нелокальными свойствами. Для модели димеров и модели LERW это следует из определения, в то время как нелокальность 
ASM следует из критерия рекуррентности [22], который требует сканирования всей конфигурации высот, прежде чем отнести ее к рекуррентному классу. Эти нелокальные свойства обычно делают конкретные вычисления особенно сложными. Следует также упомянуть проблему конформной инвариантности, которая обсуждалась в терминах конформной теории поля для моделей димеров [24]-[26], покрывающих сетей [27] и для ASM [28]-[31], а также в терминах стохастического процесса Левнера в случаях LERW и покрывающих деревьев [32], [33].

Цель данной статьи - проследить вычисление констант, упомянутых в аннотации. Мы расскажем, как возникло предположение о рациональности средней высоты в ASM и как оно трансформировалось в процессе дальнейших исследований. Окончательное вычисление [14] основано на обнаруженной в работе [34] связи между вероятностью возврата LERW и средней высотой ASM: $P_{\text {ret }}=\langle h\rangle / 2-5 / 4$. Оказалось, что эти две константы выражаются, в свою очередь, через три других константы, недавно введенных Левиным и Пересом [2], а именно через петлевую константу $\xi$ квадратной решетки $\mathbb{Z}^{2}$, отношение $\tau$ между числами покрывающих деревьев и унициклов и среднюю длину цикла $\lambda$ в покрывающем уницикле при условии, что все эти величины определены в пределе бесконечной квадратной решетки. Кроме того, все эти константы имеют $d$-мерные аналоги и связаны между собой рациональными отношениями в любой размерности [2]. Следовательно, вычислив вероятность возврата LERW $P_{\text {ret }}$, мы одновременно получим результаты для искомых констант.

B разделах 2 и 3 мы дадим выражения для вероятности возврата LERW и средней высоты ASM в терминах покрывающих деревьев, обладающих определенными свойствами. В разделе 4 мы изложим результаты Левина и Переса [2]. Раздел 5 содержит доказательство гипотезы $P_{\text {ret }}=5 / 16$ путем сведения задачи о покрывающих деревьях определенной конфигурации к задаче о мономерах и димерах. Необходимо отметить, что это доказательство не требует вычисления многомерных интегралов, полученных в работе [35]. Доказательство является чисто комбинаторным и показывает, как вычисление покрывающих графов с определенными нелокальными свойствами может быть сведено к вычислению локальных мономер-димерных корреляционных функций, что является существенно более простой задачей. Таким образом удается избежать сложных графических конструкций, которые использовались в работах [30], [35]. Кроме упрощения задачи, наше доказательство показывает, почему вероятность возврата LERW и связанные с ней константы принимают рациональные значения.

\section{2. СРЕДНЯЯ ВЫСОТА ASM}

История, изложенная ниже, начинается с вычисления вероятности высот ASM. ASM конечного объема [21] определяется через переменные $h_{n}$ высот в каждом узле конечной двумерной квадратной решетки. Переменные $h_{n}$ принимают в устойчивых конфигурациях значения $1,2,3,4$. Частицы добавляются по одной в случайно выбранный узел, увеличивая на единицу высоту в данном узле. Во многих статьях по ASM используются переменные $\sigma_{n}=h_{n}-1$ вместо переменных высот. Если высота превосходит 4, данный узел становится неустойчивым и релаксирует, передавая по одной частице в каждый соседний узел. На границе частицы могут покидать решетку. После релаксации данного узла один или несколько соседних узлов могут оказаться неустойчивыми и также релаксировать, таким образом неустойчивость 
передается от соседа к соседу. Когда на решетке не остается ни одного неустойчивого узла, в случайно выбранный узел добавляется новая частица и релаксационный процесс продолжается.

Анализ этой дискретной динамики был проведен Дхаром [22], который определил признаки рекуррентных конфигураций. Он показал, что система входит в подмножество рекуррентных конфигураций за конечное время, которое определяется начальными условиями, и затем никогда не покидает его. Поэтому вероятностное распределение, возникающее в асимптотическом пределе, обращается в нуль для нерекуррентных конфигураций. Можно показать, что это распределение однородно на рекуррентном подмножестве, размер которого $\sim 3.21^{L^{2}}$ для решетки $L \times L$. Дхар нашел также критерий отбора рекуррентных конфигураций. Его конкретная форма не используется нами в дальнейших рассуждениях.

Поскольку конфигурации ASM представлены переменными $\{1,2,3,4\}$ и распределены однородно в рекуррентном подмножестве, возникает естественный вопрос о статистических свойствах этих переменных и их пространственных корреляциях. В частности, одна из первых задач - это найти распределение высот в одном узле. Чтобы упростить задачу и избежать рассмотрения граничных эффектов, мы рассмотрим узел далеко от границы и устремим объем в бесконечность; так, $P_{i}$, $i=1,2,3,4$, будет означать вероятность найти высоту $i$ в фиксированном узле в пределе бесконечной решетки. Первая из четырех вероятностей была получена Мажумдаром и Дхаром [36],

$$
P_{1}=\frac{2}{\pi^{2}}-\frac{4}{\pi^{3}}
$$

Три других вероятности $P_{2}, P_{3}$ и $P_{4}$ оказываются более сложными для вычисления в терминах покрывающих деревьев. Алгоритм сжигания [23], предложенный Дхаром [22] как метод установления рекуррентности, позволяет установить однозначное соответствие между множеством рекуррентных конфигураций на решетке и множеством корневых (ориентированных) покрывающих деревьев на той же решетке. Свойства покрывающих деревьев, соответствующих определенной высоте в данном узле, были установлены в работе [35]. Выяснилось, что доля конфигураций $P_{i}$, имеющих высоту $i$ в фиксированном узле, выражается через следующие отношения для покрывающих деревьев:

$$
P_{1}=\frac{X_{0}}{4 \mathcal{N}}, \quad P_{2}=P_{1}+\frac{X_{1}}{3 \mathcal{N}}, \quad P_{3}=P_{2}+\frac{X_{2}}{2 \mathcal{N}}, \quad P_{4}=P_{3}+\frac{X_{3}}{\mathcal{N}}
$$

где $X_{k}, k=0,1,2,3$, - это число покрывающих деревьев таких, что данный узел имеет точно $k$ предшественников среди своих ближайших соседей [35] (узел $x$ называется предшественником узла $y$, если путь по ветвям дерева из точки $x$ в корень проходит через $y$ ). Величина $\mathcal{N}$ - это полное число покрывающих деревьев на решетке. Равенства (2.2) справедливы для конечной решетки. Переход в них к бесконечному объему допустим и определяет искомые вероятности. В отличие от $X_{0}$, величины $X_{k}$ при $k \geqslant 1$ определяются более сложно, потому что они требуют перечисления покрывающих деревьев, обладающих нелокальными свойствами (свойства деревьев, определяющих $X_{0}$, остаются локальными). Значения всех этих величин, 
полученных в [35], приводят к следующим результатам:

$$
\begin{aligned}
& P_{2}=\frac{1}{2}-\frac{3}{2 \pi}-\frac{2}{\pi^{2}}+\frac{12}{\pi^{3}}+\frac{I_{1}}{4}, \\
& P_{3}=\frac{1}{4}+\frac{3}{2 \pi}+\frac{1}{\pi^{2}}-\frac{12}{\pi^{3}}-\frac{I_{1}}{2}-\frac{3 I_{2}}{32}, \\
& P_{4}=\frac{1}{4}-\frac{1}{\pi^{2}}+\frac{4}{\pi^{3}}+\frac{I_{1}}{4}+\frac{3 I_{2}}{32},
\end{aligned}
$$

где $I_{\nu}, \nu=1,2$, являются сингулярными четырехкратными интегралами

$$
I_{\nu}=\frac{1}{(2 \pi)^{4}} \iiint \int_{0}^{2 \pi} \frac{i \sin \beta_{1} \operatorname{det} M_{\nu} d \alpha_{1} d \alpha_{2} d \beta_{1} d \beta_{2}}{D\left(\alpha_{1}, \beta_{1}\right) D\left(\alpha_{2}, \beta_{2}\right) D\left(\alpha_{1}+\alpha_{2}, \beta_{1}+\beta_{2}\right)},
$$

где $D(\alpha, \beta)=2-\cos \alpha-\cos \beta$, а матрицы $M_{1}$ и $M_{2}$ имеют вид

$$
\begin{aligned}
M_{1} & =\left(\begin{array}{cccc}
1 & 1 & e^{i \alpha_{2}} & 1 \\
3 & e^{i\left(\beta_{1}+\beta_{2}\right)} & e^{i\left(\alpha_{2}-\beta_{2}\right)} & e^{-i \beta_{1}} \\
4 / \pi-1 & e^{i\left(\alpha_{1}+\alpha_{2}\right)} & 1 & e^{-i \alpha_{1}} \\
4 / \pi-1 & e^{-i\left(\alpha_{1}+\alpha_{2}\right)} & e^{2 i \alpha_{2}} & e^{i \alpha_{1}}
\end{array}\right) \\
M_{2} & =\left(\begin{array}{ccc}
e^{i \beta_{2}} & e^{-i\left(\alpha_{1}+\alpha_{2}\right)-i\left(\beta_{1}+\beta_{2}\right)} & e^{i \beta_{1}} \\
e^{-i \alpha_{2}} & 1 & e^{-i \alpha_{1}} \\
e^{i \alpha_{2}} & e^{-2 i\left(\alpha_{1}+\alpha_{2}\right)} & e^{i \alpha_{1}}
\end{array}\right)
\end{aligned}
$$

Первые численные оценки дали следующие результаты для вероятностей: $P_{2} \simeq$ $0.1739, P_{3} \simeq 0.3063$ и $P_{4} \simeq 0.4461$. Вскоре после вывода интегралов (2.4) Питер Грасбергер предпринял более аккуратную оценку $P_{2}, P_{3}, P_{4}$ и выдвинул замечательную гипотезу (см. [37]) о том, что средняя высота имеет неожиданный рациональный вид:

$$
\langle h\rangle=P_{1}+2 P_{2}+3 P_{3}+4 P_{4}=\frac{25}{8} .
$$

Через несколько лет вычисление $P_{i}$ на плоскости и полуплоскости было воспроизведено в работе [30], в которой было получено точное соотношение между $P_{2}$ и $P_{3}$ :

$$
(\pi-8) P_{2}+2(\pi-2) P_{3}=\pi-2-\frac{3}{\pi}+\frac{12}{\pi^{2}}-\frac{48}{\pi^{3}} .
$$

Авторы работы [30] выделили из $I_{1}$ другой интеграл $J_{2}$,

$$
I_{1}=\frac{2}{\pi^{2}}(\pi-2)+\left(\frac{4}{\pi}-2\right) J_{2},
$$

и вычислили $J_{2}$ с точностью до двенадцатого знака. Впоследствии точность была увеличена до 25 знаков. Эти результаты привели к предположению $J_{2}=1 / 2$ и к следующим выражениям для вероятностей высот:

$$
P_{2}=\frac{1}{4}-\frac{1}{2 \pi}-\frac{3}{\pi^{2}}+\frac{12}{\pi^{3}}, \quad P_{3}=\frac{3}{8}+\frac{1}{\pi}-\frac{12}{\pi^{3}}, \quad P_{4}=\frac{3}{8}-\frac{1}{2 \pi}+\frac{1}{\pi^{2}}+\frac{4}{\pi^{3}} .
$$

Выдвинутая около 20 лет тому назад гипотеза о средней высоте 25/8 оставалась загадочным фактом теории ASM из-за своей удивительной простоты. Численный результат $25 / 8 \pm 10^{-25}$ снял все сомнения относительно рациональности $\langle h\rangle$, но строгое математическое доказательство отсутствовало. Ниже мы докажем эту гипотезу, но не путем вычисления интегралов, дающих среднюю высоту, а с помощью недавних наблюдений относительно вероятности возврата LERW. 

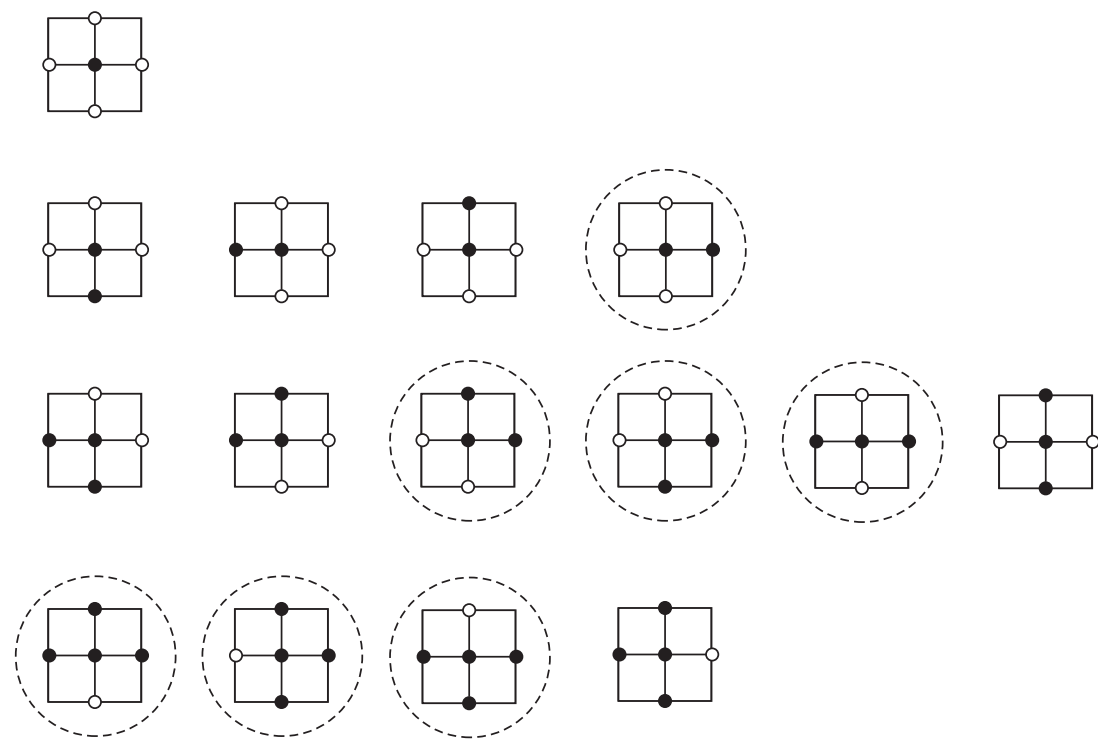

Рис. 1. Все возможные расположения предшественников на ближайших соседних узлах квадратной решетки.

\section{3. ВЕРОЯТНОСТЬ ВОЗВРАTA LERW И СРЕДНЯЯ ВЫСОТА АSM}

Для LERW, начинающегося в начале координат, мы определили вероятность возврата $P_{\text {ret }}$ как вероятность того, что путь проходит через фиксированный соседний узел начального узла, скажем правый соседний узел. В терминах однородно распределенных покрывающих деревьев $P_{\text {ret }}$ это вероятность того, что начало координат является предшественником своего правого ближайшего соседа. Рассмотрим проблему предшественников для всех ближайших соседей данного узла. Сначала фиксируем узел $i_{0}$ на квадратной решетке. Пусть $j_{0}$ - правый ближайший сосед этого узла. Далее рассмотрим четыре возможных случая, когда $i_{0}$ имеет точно $k$ предшественников среди ближайших соседей, $k=0,1,2,3$ (см. рис. 1). Согласно определению $X_{i}$ в (2.2) верхняя диаграмма представляет $X_{0}$, вторая строка диаграмм $-X_{1}$, третья $-X_{2}$, четвертая $-X_{3}$. Очевидно, что при $k=0$ вершина $j_{0}$ не является предшественником $i_{0}$. При $k=1$ мы имеем одну из четырех эквивалентных ситуаций, когда $j_{0}$ - это предшественник $i_{0}$. При $k=3$ мы имеем три из четырех эквивалентных ситуаций, когда $j_{0}$ является предшественником. Случай $k=2$ требует отдельного рассмотрения. Здесь мы имеем шесть конфигураций, которые не являются эквивалентными. Мы можем выделить две группы эквивалентных конфигураций: первые четыре и две последние конфигурации в третьей строке рис. 1 . Мы снова отбираем ситуации, в которых $j_{0}$ является предшественником $i_{0}$. Это две выделенные конфигурации в первой группе и одна во второй группе. Таким образом, заданному условию удовлетворяет половина диаграмм третьей строки рис. 1. Теперь мы можем взять линейную комбинацию $X_{1}, X_{2}$ и $X_{3}$ с коэффициентами $1 / 4,1 / 2$ и $3 / 4$, чтобы получить искомую вероятность $P\left(i_{0}, j_{0}\right)$ того, что $j_{0}$ является 
предшественником $i_{0}$ :

$$
P\left(i_{0}, j_{0}\right)=P_{\text {ret }}=\frac{X_{1}}{4 \mathcal{N}}+\frac{X_{2}}{2 \mathcal{N}}+\frac{3 X_{3}}{4 \mathcal{N}}
$$

Используя равенство (2.2) для того, чтобы извлечь $X_{k}$ из $P_{i}$, а также равенство $P_{1}+P_{2}+P_{3}+P_{4}=1$, мы выразим вероятность возврата LERW через среднюю высоту ASM:

$$
P_{\text {ret }}=\frac{1}{4}\left(-3 P_{1}-P_{2}+P_{3}+3 P_{4}\right)=\frac{1}{2}\left(P_{1}+2 P_{2}+3 P_{3}+4 P_{4}\right)-\frac{5}{4}=\frac{\langle h\rangle}{2}-\frac{5}{4} .
$$

\section{4. НЕКОТОРЫЕ НОВЫЕ КОНСТАНТЫ}

Левин и Перес [2] определили петлевую константу $\xi$ квадратной решетки $\mathbb{Z}^{2}$ как ожидаемое число соседей начала координат $(0,0)$ для бесконечного LERW, начинающегося в точке $(0,0)$. Из-за симметрии квадратной решетки петлевая константа и вероятность возврата связаны соотношением

$$
\xi=4 P_{\text {ret }}
$$

Одним из замечательных фактов теории ASM является теорема, доказанная Мерино [38]. Полиномом Тутта конечного графа $G=(V, E)$ с множеством вершин $V$ и множеством ребер $E$ называется полином

$$
T_{G}(x, y)=\sum_{A \subset E}(x-1)^{c(A)-1}(y-1)^{c(A)+|A|-|E|},
$$

где $c(A)$ - это число связных компонент покрывающего подграфа $(V, A)$. Теорема Мерино гласит:

$$
T_{G}(1, y)=y^{\delta-|E|} \sum_{\sigma} y^{|\sigma|}
$$

где суммирование проводится по всем рекуррентным состояниям $\sigma$ ASM на графе $G$ с поглощающей вершиной $s,|\sigma|=\sum_{n \neq s} \sigma_{n}$ - полное число частиц, а $\delta$ - степень поглощающей вершины $s$. Используя теорему Мерино, Левин и Перес ввели новую константу $\tau$, которая получается делением числа покрывающих унициклов графа $G$ на число покрывающих деревьев. В пределе $G \rightarrow \mathbb{Z}^{2}$ эта константа равна

$$
\tau=\frac{\xi-1}{2}=2 P_{\text {ret }}-\frac{1}{2} .
$$

Для средней длины $\lambda$ цикла в USU на $\mathbb{Z}^{2}$ Левин и Перес получили

$$
\lambda=\frac{1}{\tau} .
$$

Таким образом, все четыре константы $\xi, \tau, \lambda$ и $\langle h\rangle$ оказываются связанными с вероятностью возврата $P_{\text {ret }}$.

Для нахождения $P_{\text {ret }}$ мы должны рассмотреть все возможные траектории LERW, начинающиеся в начале координат и проходящие через правый соседний узел. Эти 


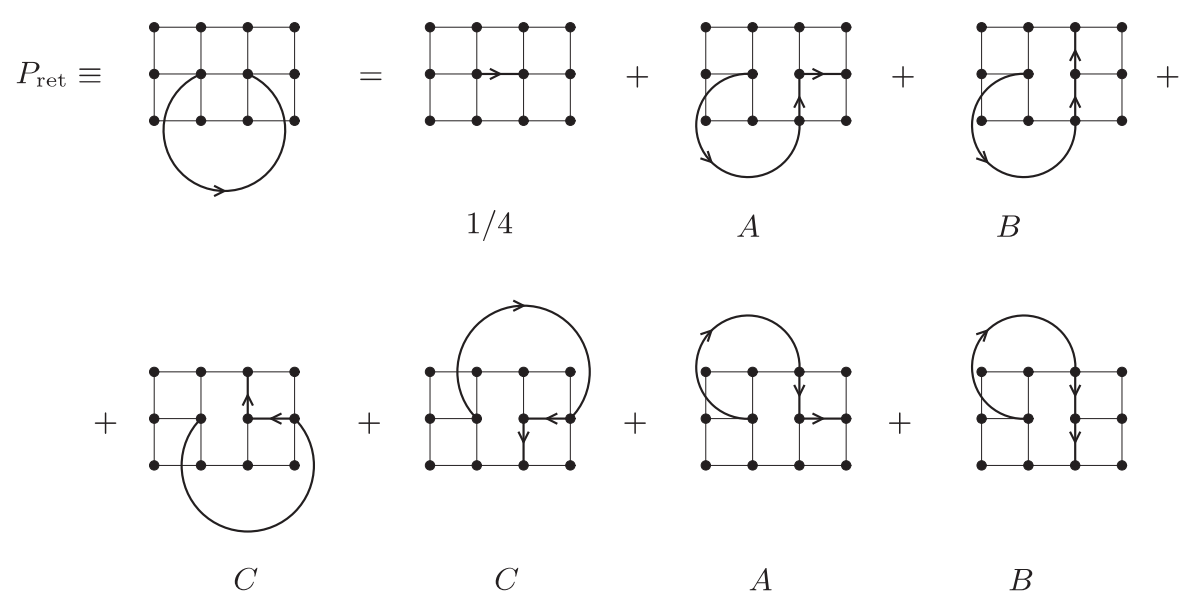

Рис. 2. Разложение вероятности возврата в терминах химических путей между двумя соседними вершинами.

траектории, являющиеся химическими путями на покрывающем дереве, можно разделить на семь групп, показанных на рис. 2. Некоторые из этих групп эквивалентны из-за симметрии решетки.

Обозначая через $A, B, C$ соответствующие доли покрывающих деревьев каждой группы, мы получим

$$
P_{\text {ret }}=\frac{1}{4}+2(A+B+C) .
$$

Остается вычислить $A, B$ и $C$. Это можно сделать, рассматривая специальные расположения мономеров и димеров.

\section{5. МОНОМЕР-ДИМЕРНЫЕ РАСЧЕТЫ И ВЕРОЯТНОСТЬ ВОЗВРАTA LERW}

Рассмотрим квадратную $[(2 n-1) \times(2 n-1)]$-решетку $\mathcal{L}$ с удаленной нижней правой вершиной. Вершины решетки можно разделить на три подмножества: 1) черные вершины, образующие подрешетку $\mathcal{B}$ узлов с нечетно-нечетными координатами; 2) белые вершины, образующие подрешетку $\mathcal{W}$ узлов с четно-четными координатами; 3) серые вершины - оставшиеся вершины решетки. Удаленную угловую вершину, в которую будет помещен корень возникающего дерева, обозначим через $r$. Рассмотрим плотную упаковку димеров на решетке $\mathcal{L}$. Каждый димер покрывает два узла, черный и серый или белый и серый. Соответствие, предложенное Темперли, связывает два множества стрелок с множеством димерных конфигураций следующим образом. Заменим каждый димер, покрывающий черный узел, стрелкой, направленной от этого узла к серому узлу, покрываемому этим димером. Подобным образом поступаем с димерами, покрывающими белые узлы. Множества стрелок из черных и белых узлов образуют два множества ациклических конфигураций стрелок, которые образуют покрывающие деревья на двух подрешетках $\mathcal{B}$ и $\mathcal{W}$ (см. рис. 3). Эти два покрывающих дерева являются дуальными; из каждого из них может быть восстановлена димерная конфигурация. Направленный путь от 


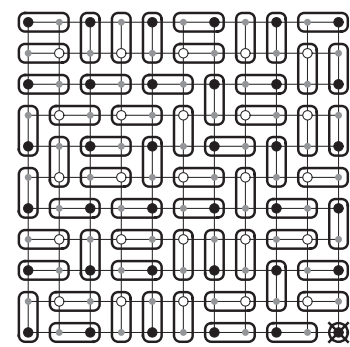

a

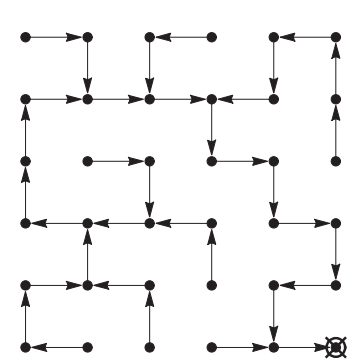

6

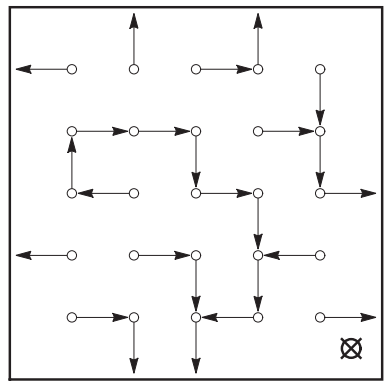

B

Рис. 3. Димерная упаковка (а); покрывающее дерево на нечетно-нечетной подрешетке (б) и на четно-четной (в) подрешетке.



a

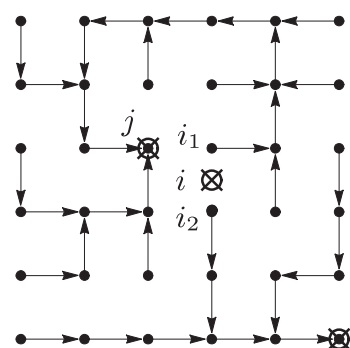

6



B

Рис. 4. Димерное покрытие с двумя мономерами (а); покрывающие деревья в случае I (б, в).

узла $x \in \mathcal{B}(x \in \mathcal{W})$ к узлу $y \in \mathcal{B}(y \in \mathcal{W})$ вдоль ветвей черного (белого) покрывающего дерева является химическим путем от $x$ к $y$. Как показано в [1], [17]-[20], статистические свойства химического пути совпадают со свойствами LERW. Hac интересует прежде всего вероятность возврата, т. е. вероятность того, что химический путь, начинающийся в данной точке покрывающего дерева, посетит один из соседних узлов этой точки, например правый узел, прежде чем достигнет корня, помещенного в точке $r$.

Рассмотрим теперь плотную димерную упаковку на решетке $\mathcal{L}^{\prime}$, полученной из решетки размера $(2 n-1) \times(2 n-1)$ удалением трех узлов: углового узла $r$, серого узла $i$ и черного узла $j$. У серого узла $i$ имеются два ближайших соседа $i_{1}, i_{2} \in \mathcal{B}$ (см. рис. 4). Кеньон заметил (лемма 17 в [39]), что если бы узел $j$ был на границе $\mathcal{L}^{\prime}$, то узлы $i_{1}$ и $i_{2}$ принадлежали бы различным компонентам двухкомпонентного покрывающего дерева на черной подрешетке: одна компонента с корнем $r$, а другая с корнем $j$. Действительно, $i_{1}$ и $i_{2}$ не могут быть в одной компоненте, поскольку в этом случае химический путь от $i_{1}$ до $i_{2}$ вместе с ребром из $\mathcal{B}$, соединяющим $i_{1}$ и $i_{2}$, образовали бы замкнутый контур, окружающий нечетное число узлов решетки $\mathcal{L}^{\prime}$, которые не могут быть покрыты димерами. 


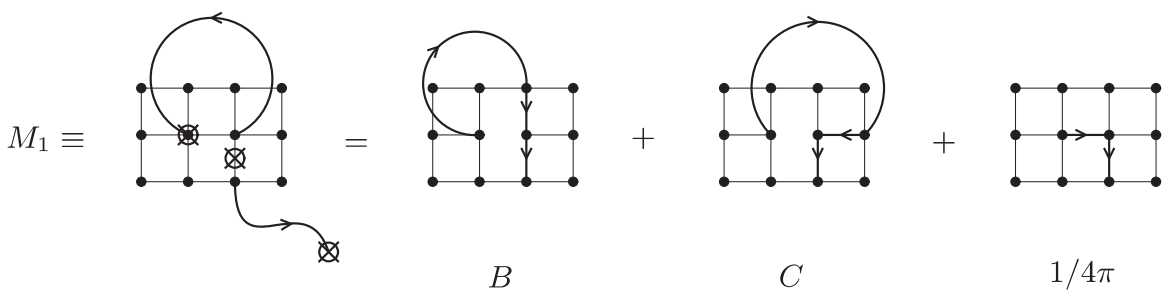

Рис. 5. Три возможных конфигурации путей в случае I.

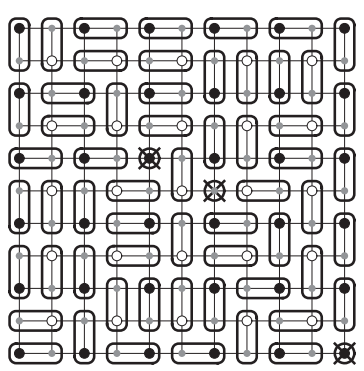

a

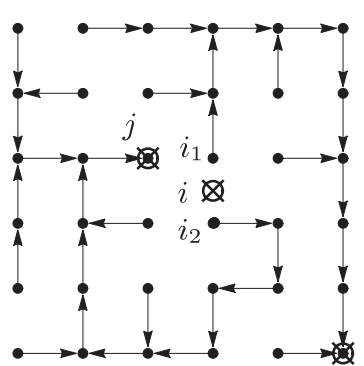

б

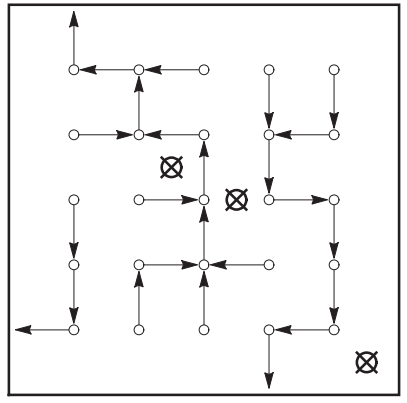

B

Рис. 6. Димерное покрытие и покрывающие деревья в случае II.

Если $j$ не находится на границе, это рассуждение больше не справедливо, потому что пути из $i_{1}$ и $i_{2}$ могут образовать петлю вокруг $j$ или оба устремиться в корень $r$, охватывая $j$. Мы выберем точку $j$ в левом ближайшем к $i_{1}$ соседнем узле на подрешетке $\mathcal{B}$. Тогда замкнутая петля вокруг $j$ невозможна из-за отсутствия узлов между $j$ и $i_{1}$ или $i_{2}$ на подрешетке $\mathcal{B}$. Остаются три возможности.

I. Первая возможность изображена на рис. 4б: путь из $i_{1}$ направляется в $j$, а путь из $i_{2}-$ в $r$. Обращая ориентацию первого пути и вставляя стрелку от $i_{1} \mathrm{k} i_{2}$, мы получим путь из $j$ в $r$, проходящий через ребро $\left(i_{1}, i_{2}\right)$. В зависимости от выбора ребер в окрестности узла $i_{1}$ мы получим три конфигурации пути, изображенные на рис. 5. Первые две из них совпадают с конфигурациями, обозначенными в разделе 4 как $B$ и $C$ (с точностью до зеркального отражения). Третья конфигурация соответствует тем покрывающим деревьям на $\mathcal{B}$, которые содержат ребра $\left(j, i_{1}\right)$ и $\left(i_{1}, i_{2}\right)$. $\mathrm{B}$ терминах димеров на исходной решетке $\mathcal{L}$ это соответствует димерной упаковке $\mathrm{c}$ двумя перпендикулярными димерами, покрывающими узлы $j$ и $i_{1}$. Фракция таких упаковок представляет собой локальную димер-димерную корреляционную функцию, вычисленную Фишером и Стивенсоном в [9] и равную $1 / 4 \pi$ в пределе большой решетки.

II. Путь из $i_{1}$ направляется в $r$, а путь из $i_{2}-$ в $j$ (см. рис. 6 ). Подобно случаю I, мы обращаем ориентацию второго пути и вставляем стрелку от $i_{2}$ к $i_{1}$, чтобы получить путь из $j$ в $r$, проходящий через ребро $\left(i_{2}, i_{1}\right)$. Это приводит к двум локальным конфигурациям путей $A$ и $B$, показанным на рис. 7 .

III. Узлы $i_{1}$ и $i_{2}$ принадлежат одной компоненте с корнем в $r$, а химический путь из этих узлов окружает компоненту с корнем в $j$ (см. рис. 8 и рис. 9). 




Рис. 7. Две возможные конфигурации путей в случае II.

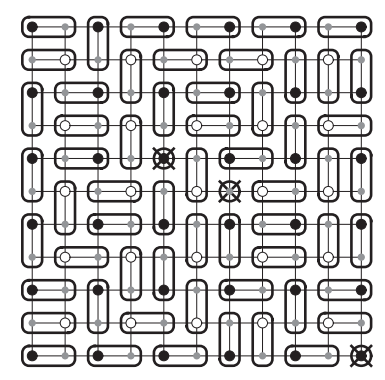

a



6

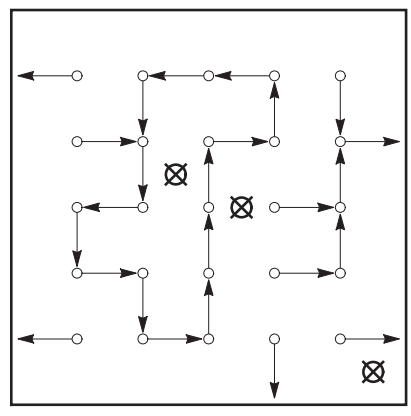

B

Рис. 8. Димерное покрытие и покрывающие деревья в случае III.

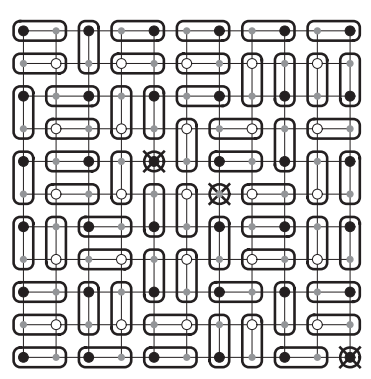

a

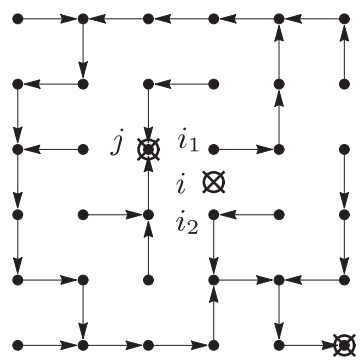

6

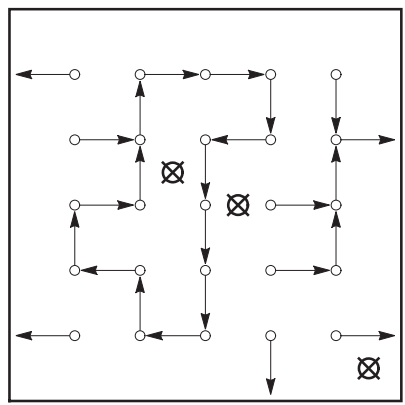

B

Рис. 9. То же, что на рис. 8 , но с противоположной ориентацией петли.

Это означает, что дуальный граф на $\mathcal{W}$ содержит одну петлю вокруг узла $j$. Эта петля единственна из-за отсутствия узлов между $i$ и $j$. Этот граф может иметь две ориентации, как показано на рис. 8 и рис. 9. Поворачивая на $\pi / 2$ вертикальную стрелку на дуальном графе, принадлежащую петле и находящуюся между $i$ и $j$, мы получим набор путей типа $B$ и $C$, но уже на подрешетке $\mathcal{W}$ (рис. 10). Число таких путей должно быть удвоено за счет двух эквивалентных ориентаций стрелок на петле. 


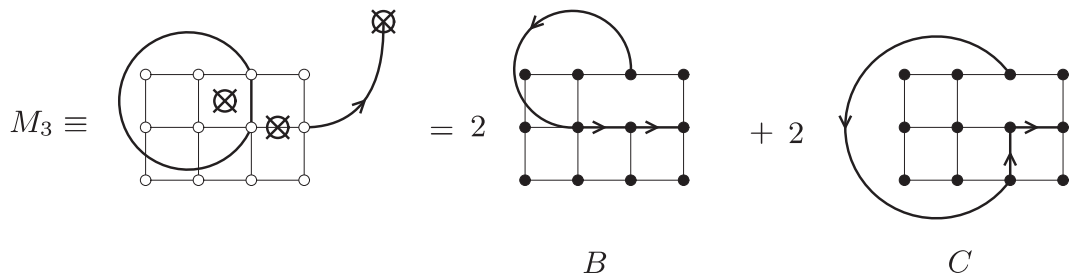

Рис. 10. Конфигурации петель в случае III.

Собирая вклады от случаев I, II, и III, мы определим, как димерные конфигурации на $\mathcal{L}^{\prime}$ связаны с классами $A, B, C$ покрывающих деревьев на подрешетке $\mathcal{B}$. Разделив эти вклады на полное число димерных конфигураций $\mathcal{L}$, мы получим, что коррелятор $P_{\operatorname{mm}}$ двух мономеров в точках $i$ и $j$ выражается через относительное число покрывающих деревьев типа $A, B, C$ в пределе большой решетки:

$$
P_{\mathrm{mm}}=M_{1}+M_{2}+M_{3}=\frac{1}{4 \pi}+A+4 B+3 C .
$$

Благодаря технике, развитой в работе [9], вычисление коррелятора мономеров легко осуществляется и дает результат $P_{\mathrm{mm}}=1 / 2 \pi$. Это дает первое соотношение для трех неизвестных $A, B, C$.

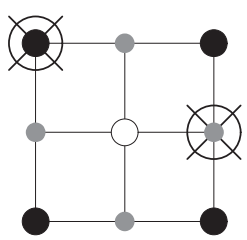

a

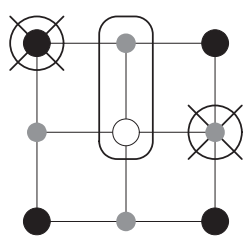

б



B

Рис. 11. Два мономера (а); два мономера и димер (б); два димера, эквивалентные случаю, приведенному на рис. 116 (в).

Для того чтобы найти второе соотношение, мы повторим предыдущий расчет, в котором, кроме двух мономеров, мы фиксируем положение димера между ними, как показано на рис. 11б. Таким образом, вместо мономер-мономерной корреляционной функции, мы рассмотрим корреляцию мономер-димер-мономер. При таком расположении мономеров и димера запрещены все ориентации стрелок, кроме случая I и одной ориентации петли в случае III. Следовательно, мы можем написать

$$
P_{\mathrm{mdm}}=M_{2}+\frac{1}{2} M_{3}=A+2 B+C \text {. }
$$

Коррелятор $P_{\mathrm{mdm}}$ эквивалентен двум фиксированным димерам, как показано на рис. 11в. Снова используя результаты работы [9], мы получим $P_{\operatorname{mdm}}=1 / 8-1 / 4 \pi$. Наконец, мы заметим, что равенство $A=B$ следует из двух преобразований, показанных на рис. 12: мы обращаем ориентацию петли, перемещаем вертикальную стрелку, направленную вверх, в положение горизонтальной стрелки, направленной

7 Теоретическая и математическая физика, т. 187, № 3, 2016 г. 


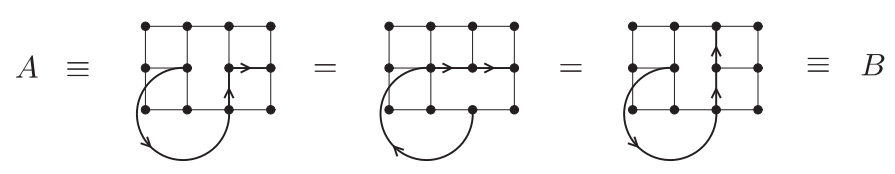

Рис. 12. Два преобразования, доказывающие эквивалентность $A$ и $B$.

вправо, и затем производим зеркальное отражение. Два уравнения (5.1) и (5.2) легко решаются и приводят к результату

$$
A=B=\frac{3}{32}-\frac{1}{4 \pi}, \quad C=\frac{1}{2 \pi}-\frac{5}{32} .
$$

Подставляя эти значения в выражение для вероятности возврата (4.6), мы получаем $P_{\text {ret }}=5 / 16$ и $\langle h\rangle=25 / 8$.

Приведем полный список доказанных констант:

$$
P_{\text {ret }}=\frac{5}{16}, \quad\langle h\rangle=\frac{25}{8}, \quad \xi=\frac{5}{4}, \quad \tau=\frac{1}{8}, \quad \lambda=8 .
$$

\section{6. НЕДАВНИЕ ИССЛЕДОВАНИЯ}

Почти одновременно со статьей [14] Кеньон и Вилсон опубликовали подробную работу [40] о вычислениях UST для различных топологий графов, определенных на двумерных поверхностях. Они разработали технику вычислений решеточных функций Грина на поверхностях, топологически эквивалентных кольцам с линиями дефектов и линиями, соединяющими различные точки на границах колец. Эта мощная техника позволяет вычислять более сложные корреляционные функции, чем вычисленные выше. Например, в рамках этой техники можно вычислить петлевые константы $\xi_{\mathrm{t}}$ и $\xi_{\mathrm{h}}$ для треугольной и гексагональной решеток. Кеньон и Вильсон получили $\xi_{\mathrm{t}}=5 / 18$ и $\xi_{\mathrm{h}}=13 / 36$. Позже Кассель, Кеньон и Ву [41] рассмотрели свойства цикла в USU более детально. В дополнение к средней длине цикла (4.5) они вычислили асимптотики первого и второго моментов для распределения площади цикла на графе $G_{n} \subset \mathbb{Z}^{2}$ такого, что $G_{n} / n$ является аппроксимацией ограниченной области $D \subset \mathbb{C}$. Они доказали, что первый и второй моменты растут как $(4 / \pi) \ln n$ и $C \cdot \operatorname{Area}(D) n^{2}$, где $C$ - константа, зависящая от $D$.

Недавно Сан и Вильсон [42] рассмотрели среднюю плотность ASM $\rho$ на случайном планарном графе. Используя так называемое соответствие "гамбургер-чизбургер" для построения корневых планарных покрытий с $n$ ребрами, они доказали, что $\rho=5 / 2$ в пределе $n \rightarrow \infty$.

После всех этих косвенных вычислений средней высоты Карачиоло и Спортиелло [43] нашли прямой путь вычисления интегралов (2.6). Они заметили, что эти интегралы имеют форму двухпетлевых фейнмановских интегралов двумерной скалярной теории поля на решетке. Используя удобные символические обозначения, они свели двухпетлевые решеточные интегралы к квадратичным полиномам однопетлевых интегралов. Таким образом, теорема о рациональных значениях констант и, в частности значение $\langle h\rangle=25 / 8$ получили красивое аналитическое подтверждение.

Благодарности. Авторы благодарны акад. Ю. Г. Шукуряну и проф. Ф. Рюэллю за плодотворное сотрудничество. 


\section{Список литературы}

[1] R. Pemantle, Ann. Probab., 19:4 (1991), 1559-1574.

[2] L. Levine, Y. Peres, Random Structures Algorithms, 45:1 (2014), 1-13.

[3] G. Kirchhoff, Ann. Phys., 148:12 (1847), 497-508.

[4] R. H. Fowler, G. S. Rushbrooke, Trans. Faraday Soc., 33 (1937), 1272-1294.

[5] P. W. Kasteleyn, Physica, 27:12 (1961), 1209-1225.

[6] P. W. Kasteleyn, J. Math. Phys., 4:2 (1963), 287-293.

[7] M. E. Fisher, Phys. Rev., 124:6 (1961), 1664-1672.

[8] H. N. V. Temperley, M. E. Fisher, Phil. Mag., 6:68 (1961), 1061-1063.

[9] M. E. Fisher, J. Stephenson, Phys. Rev., 132:4 (1963), 1411-1431.

[10] R. Kenyon, "Lectures on dimers", Statistical Mechanics, IAS/Park City Mathematics Series, 16, eds. S. Sheffield, T. Spencer, AMS, Providence, RI, 2009, 191-230, arXiv: 0910.3129.

[11] H. N. V. Temperley, Combinatorics. The Proceedings of the British Combinatorial Conference (University College of Wales, Aberystwyth, 2-6 July, 1973), London Mathematical Society Lecture Note Series, 13, eds. T.P. McDonough, V. C. Mavron, Cambridge Univ. Press, London-New York, 1974, 202-204.

[12] R. Burton, R. Pemantle, Ann. Probab., 21:3 (1993), 1329-1371.

[13] J. Bouttier, M. Bowick, E. Guitter, M. Jeng, Phys. Rev. E, 76:4 (2007), 041140, 15 pp., arXiv: 0706.1016.

[14] V.S. Poghosyan, V. B. Priezzhev, P. Ruelle, J. Stat. Mech., 2011:10 (2011), P10004, 12 pp., arXiv: 1106.5453.

[15] G. F. Lawler, Duke Math. J., 47:3 (1980), 655-693.

[16] J. G. Propp, D. B. Wilson, J. of Algorithms, 27:2 (1998), 170-217.

[17] A. Z. Broder, "Generating random spanning trees", 30th IEEE Annual Symposium on Foundations of Computer Science (Research Triangle Park, North Carolina, USA, 30 October 1 November, 1989), IEEE, New York, 1989, 442-447.

[18] D. Aldous, SIAM J. Discrete Math., 3:4 (1990), 450-465.

[19] S. N. Majumdar, Phys. Rev. Lett., 68:15 (1992), 2329-2331.

[20] D. B. Wilson, "Generating random spanning trees more quickly than the cover time", Proceedings of the Twenty-eighth Annual ACM Symposium on the Theory of Computing (Philadelphia, Pennsylvania, May 22-24, 1996), ACM, New York, 1996, 296-303.

[21] P. Bak, C. Tang, K. Wiesenfeld, Phys. Rev. Lett., 59:4 (1987), 381-384.

[22] D. Dhar, Phys. Rev. Lett., 64:14 (1990), 1613-1616.

[23] S. N. Majumdar, D. Dhar, Phys. A, 185:1 (1992), 129-145.

[24] R. Kenyon, Ann. Probab., 29:3 (2001), 1128-1137.

[25] N.Sh. Izmailian, V.B. Priezzhev, P. Ruelle, C.-K. Hu, Phys. Rev. Lett., 95:26 (2005), 260602, 4 pp., arXiv: cond-mat/0512703.

[26] S. Papanikolaou, E. Luijten, E. Fradkin, Phys. Rev. B, 76:13 (2007), 134514, 28 pp., arXiv: cond-mat/0607316.

[27] J. G. Brankov, S. Y. Grigorev, V.B. Priezzhev, I. Y. Tipunin, J. Stat. Mech., 2008:11 (2008), P11017.

[28] S. Mahieu, P. Ruelle, Phys. Rev. E, 64 (2001), 066130, arXiv: 0810.2231.

[29] P. Ruelle, Phys. Lett. B, 539:1-2 (2002), 172-177, arXiv: hep-th/0203105.

[30] M. Jeng, G. Piroux, P. Ruelle, J. Stat. Mech., 2006:10 (2006), P10015.

[31] V.S. Poghosyan, S. Y. Grigorev, V. B. Priezzhev, P. Ruelle, J. Stat. Mech., 2010:7 (2010), P07025.

[32] O. Schramm, Israel J. Math., 118 (2000), 221-288.

[33] G. F. Lawler, O. Schramm, W. Werner, Ann. Probab., 32:18 (2004), 939-995.

[34] V.S. Poghosyan, V. B. Priezzhev, Acta Polytechnica, 51:2 (2011), 59-62. 
[35] V. B. Priezzhev, J. Stat. Phys., 74:4-5 (1994), 955-979.

[36] S. N. Majumdar, D. Dhar, J. Phys. A: Math. Gen., 24:7 (1991), L357-L362.

[37] D. Dhar, Phys. A, 369:1 (2006), 29-70.

[38] C. Merino, Ann. Comb., 1:3 (1997), 253-259.

[39] R. Kenyon, Acta Math., 185:2 (2000), 239-286.

[40] R. W. Kenyon, D. B. Wilson, J. Amer. Math. Soc., 28:4 (2015), 985-1030, arXiv: 1107.3377.

[41] A. Kassel, R. Kenyon, W. Wu, Random two-component spanning forests, arXiv: 1203.4858.

[42] X. Sun, D. B. Wilson, Sandpiles and unicycles on random planar maps, arXiv: 1506.08881.

[43] S. Caracciolo, A. Sportiello, Exact integration of height probabilities in the Abelian sandpile model, arXiv: 1207.6074. 